\title{
Roadmaps para implantação do teletrabalho: mapeamento sistemático e revisão da
}

\section{literatura}

\author{
Roadmaps for telework implementation: systematic mapping and literature review \\ Roadmaps para la implementación del teletrabajo: mapeo sistemático y revisión de la literatura
}

Recebido: 21/11/2021 | Revisado: 28/11/2021 | Aceito: 29/11/2021 | Publicado: 10/12/2021

Denis Alberto Batista

ORCID: https://orcid.org/0000-0002-2545-9155 Centro Estadual de Educação Tecnológica Paula Souza, Brasil

E-mail: denisbatista1@hotmail.com

Napoleão Verardi Galegale

ORCID: https://orcid.org/0000-0003-2228-9151

Centro Estadual de Educação Tecnológica Paula Souza, Brasil

E-mail:nvg@nvg.com.br

\begin{abstract}
Resumo
A busca acelerada das organizações pelo teletrabalho, especialmente motivada pela pandemia em 2020, fez com que surgissem muitas dificuldades quanto a sua implantação de forma adequada e estruturada. O objetivo desta pesquisa é identificar as abordagens e estratégias sobre a implantação do teletrabalho, notadamente, por meio de roadmaps. Tendo por base a teoria disponível na literatura internacional sobre teletrabalho, procurou-se apresentar os termos e conceitos que se referem ao universo desta modalidade de trabalho. Utilizou-se o método da revisão sistemática da literatura, cujo protocolo adotou-se o PRISMA-P. Não foi localizada uma proposta de roadmap que servisse de roteiro estratégico para a implantação do teletrabalho nas organizações. Os resultados apresentam a relevância deste tema no mundo, sendo que as suas vantagens e desvantagens é a abordagem mais presente nas pesquisas.
\end{abstract}

Palavras-chave: Teletrabalho; Trabalho remoto; Roteiro de implantação; Revisão sistemática; Sistemas produtivos.

\begin{abstract}
The quest for telework by organizations, especially motivated by the pandemic in 2020 , caused many difficulties to arise regarding its implementation in an adequate and structured way. The objective of this research is to identify the approaches and strategies on the implementation of telework, especially through roadmaps. Based on the theory available in the international literature on telework, we sought to present the terms and concepts that refer to the universe of this type of work. The method of systematic literature review was used, whose protocol was adopted as the PRISMA-P. No roadmap proposal was found that could serve as a strategic roadmap for the implementation of teleworking in organizations. The results show the relevance of this theme in the world, and its advantages and disadvantages are the most present approach in research.
\end{abstract}

Keywords: Telework; Telecommuting; Home office; Roadmap; Systematic review; Productive systems.

\section{Resumen}

La búsqueda acelerada de organizaciones para el teletrabajo, especialmente motivada por la pandemia de 2020, provocó que surgieran muchas dificultades para su implementación de manera adecuada y estructurada. El objetivo de esta investigación es identificar los enfoques y estrategias sobre la implementación del teletrabajo, especialmente mediante hojas de ruta. A partir de la teoría disponible en la literatura internacional sobre teletrabajo, se buscó presentar los términos y conceptos que hacen referencia al universo de este tipo de trabajo. Se utilizó el método de revisión sistemática de la literatura, cuyo protocolo se adoptó como PRISMA-P. No se encontró ninguna propuesta de hoja de ruta que pudiera servir como hoja de ruta estratégica para la implementación del teletrabajo en las organizaciones. Los resultados muestran la relevancia de este tema en el mundo, y sus ventajas y desventajas son el enfoque más presente en la investigación.

Palabras clave: Teletrabajo; Trabajo remoto; Hoja de ruta de implementación; Revisión sistemática; Sistemas productivos.

\section{Introdução}

O teletrabalho tornou-se extremamente relevante no mundo, dado que é uma importante alternativa para as organizações buscarem a manutenção dos empregos, a segurança dos trabalhadores e evitar a descontinuidade de negócios, 
especialmente no cenário imposto pela pandemia (FMI, 2020).

Por mais que o teletrabalho não seja recente no mundo, para muitas organizações e profissionais ainda se apresenta como uma novidade. Dado que trabalhar em casa tornou-se uma necessidade decorrente da crise sanitária mundial, este novo cenário propicia aumento da ansiedade e uma série de outras dificuldades para organizações que não possuem experiência em sua implantação (SOBRATT, 2020).

O teletrabalho foi implantado por $46 \%$ organizações durante a pandemia de 2020, de acordo com a "Pesquisa Gestão de Pessoas na Crise Covid-19", além de constatar que 67\% destas empresas apresentaram dificuldades em sua implantação (FIA, 2020).

Baruch e Yuen (2000), Watad e Will (2003) e Morgan (2004) recomendam que antes de implantar o teletrabalho, isto precisa ser muito bem avaliado pelas organizações pois este formato de trabalho não é adequado a todo tipo de emprego. É um modelo restrito a alguns contextos e demanda uma atenção especialmente nas relações interpessoais e trabalhistas.

Ao final da pandemia, acredita-se que as empresas deverão adotar um modelo híbrido, o qual intercala espaços de trabalho corporativos e home office. Desta forma, será possível se servirem das vantagens do home office e das vantagens do escritório (MIT Technology Review, 2020).

Diante deste contexto, esta pesquisa tem como objetivo identificar as principais abordagens e estratégias em como implantar o teletrabalho. Sendo assim, foi estruturada para esta pesquisa a seguinte questão direcionadora: quais as abordagens e estratégias de implantação do teletrabalho de pesquisas publicadas entre 2015 e 2021 ?

Para responder esta questão, foram definidos estes objetivos específicos: fazer a revisão sistemática da literatura; realizar a análise quantitativa, bem como a análise qualitativa dos documentos selecionados.

E a partir destes objetivos, apresentam-se as seguintes proposições:

P1 - há abordagens distintas quanto a implantação do teletrabalho.

P2 - há roadmaps propostos na literatura para apoiar as organizações em como implantar o teletrabalho.

\section{Fundamentação Teórica}

O termo teletrabalho surge como o uso de telecomunicações e computadores para substituir de forma parcial ou total o trajeto ida e volta para o trabalho, sendo esta definição relacionada ao termo telecommuting (Nilles, 1976).

Van Sell e Jacobs (1994) definem o teletrabalho por meio do uso de computadores e equipamentos de telecomunicações para trabalhar em casa ou remotamente em outro local, um ou mais dias por semana. Girard (1995) concorda com esta definição ao afirmar que é o trabalho realizado com a utilização sistemática de telecomunicações que permitem trabalhar e comunicar à distância, trocando informações e dados.

Illegems e Verbeke (2004) descrevem o teletrabalho como o trabalho executado a partir de casa, de um centro de teletrabalho, de um escritório satélite, ou de qualquer outro local fora do escritório principal por pelo menos um dia.

Rosenfield e Alves (2011) associam o termo teletrabalho ao que é realizado remotamente através de TICs (tecnologias de informação e comunicação), propiciando o alcance dos resultados do trabalho em um local diferente daquele ocupado pela pessoa que o realiza.

Na Europa é mais usual o termo telework que enfoca as atividades executadas através de tecnologia. Já nos Estados Unidos, sobressai a utilização do termo telecommuting. Entretanto, ambos se referem a um mesmo universo de organização do trabalho (Rocha \& Amador, 2018).

No conceito amplo do teletrabalho, Sakuda (2001) ressalta que, tanto na literatura nacional como na internacional, há diferentes termos para se referir à mesma coisa e um mesmo termo para se referir a diferentes especificidades. 
A definição que cobre o conceito amplo do termo teletrabalho é a realização do trabalho remotamente em qualquer lugar, por meio de TICs, possibilitando a obtenção dos resultados do trabalho (Batista, Galegale, Azevedo \& Feitosa, 2021).

Portanto, a partir de todos estes termos e conceitos que fazem parte do universo do teletrabalho, destacam-se 7 principais descritos na Tabela 1 - Principais termos sobre o Teletrabalho.

Tabela 1 - Principais termos sobre o Teletrabalho.

\begin{tabular}{lll}
\hline \multicolumn{1}{c}{ Termo } & \multicolumn{1}{c}{ Conceito } \\
\hline $\begin{array}{l}\text { Trabalho em Domicílio } \\
\text { office, home-based work ou homework) }\end{array}$ & (Home & Refere-se ao trabalho realizado na casa do trabalhador. \\
\hline $\begin{array}{l}\text { Escritórios Satélites } \\
\text { Office Center) }\end{array}$ & (Satellite & $\begin{array}{l}\text { Os trabalhadores executam o trabalho em pequenas unidades } \\
\text { espalhadas de uma empresa central. }\end{array}$ \\
\hline Telecentros & $\begin{array}{l}\text { Trabalho realizado em estabelecimentos normalmente } \\
\text { instalados próximo ao domicílio do trabalhador que oferecem } \\
\text { postos de trabalho a empregados ou várias organizações ou } \\
\text { serviços telemáticos a clientes remotos. }\end{array}$ \\
\hline Trabalho Móvel & $\begin{array}{l}\text { Trabalho longe do principal centro de trabalho e da moradia, } \\
\text { por meio de viagens de negócios, trabalho de campo, } \\
\text { instalações do cliente ou lugares públicos, como um café ou } \\
\text { em um meio de transporte. }\end{array}$ \\
\hline Empresas remotas ou off-shore & $\begin{array}{l}\text { Call-centers ou telesserviços por meio das quais firmas } \\
\text { instalam seus escritórios-satélite ou subcontratam empresas de } \\
\text { telesserviços de outras zonas do globo com mão de obra mais } \\
\text { barata. }\end{array}$ \\
\hline Modelo Híbrido & $\begin{array}{l}\text { Modalidade em que mistura espaços de trabalho corporativos e } \\
\text { trabalho remoto. }\end{array}$ \\
\hline $\begin{array}{l}\text { Acordo com o empregador para que se trabalhe algumas horas } \\
\text { fora da empresa. }\end{array}$
\end{tabular}

Fonte: Adaptado de Rosenfield e Alves (2011).

A abordagem das pesquisas da literatura internacional claramente foca nos efeitos da implantação do teletrabalho (Leite et al., 2019). Já as pesquisas disponíveis em bancos de dados nacionais abertos até 2017, apresentam que as principais abordagens são as características do teletrabalho, a gestão em si e a gestão de pessoas e saúde mental (Schleder et al., 2018).

\section{Metodologia}

Este estudo de característica qualitativa, foi desenvolvido por meio da revisão sistemática da literatura sobre pesquisas que se referem a roadmaps para implantação do teletrabalho, bem como a aplicação da análise de conteúdo.

Para esta revisão sistemática da literatura sobre roadmaps de implantação do teletrabalho, foi adotado o protocolo PRISMA-P, que foi desenvolvido como um roteiro para apoiar pesquisadores na realização de revisões sistemáticas e metaanálises que retornem um conjunto mínimo de itens importantes a serem considerados no protocolo de pesquisa (Moher et al., 2015). 
Figura 1 - Fluxograma - Revisão Sistemática sobre Roadmap de Implantação do Teletrabalho.

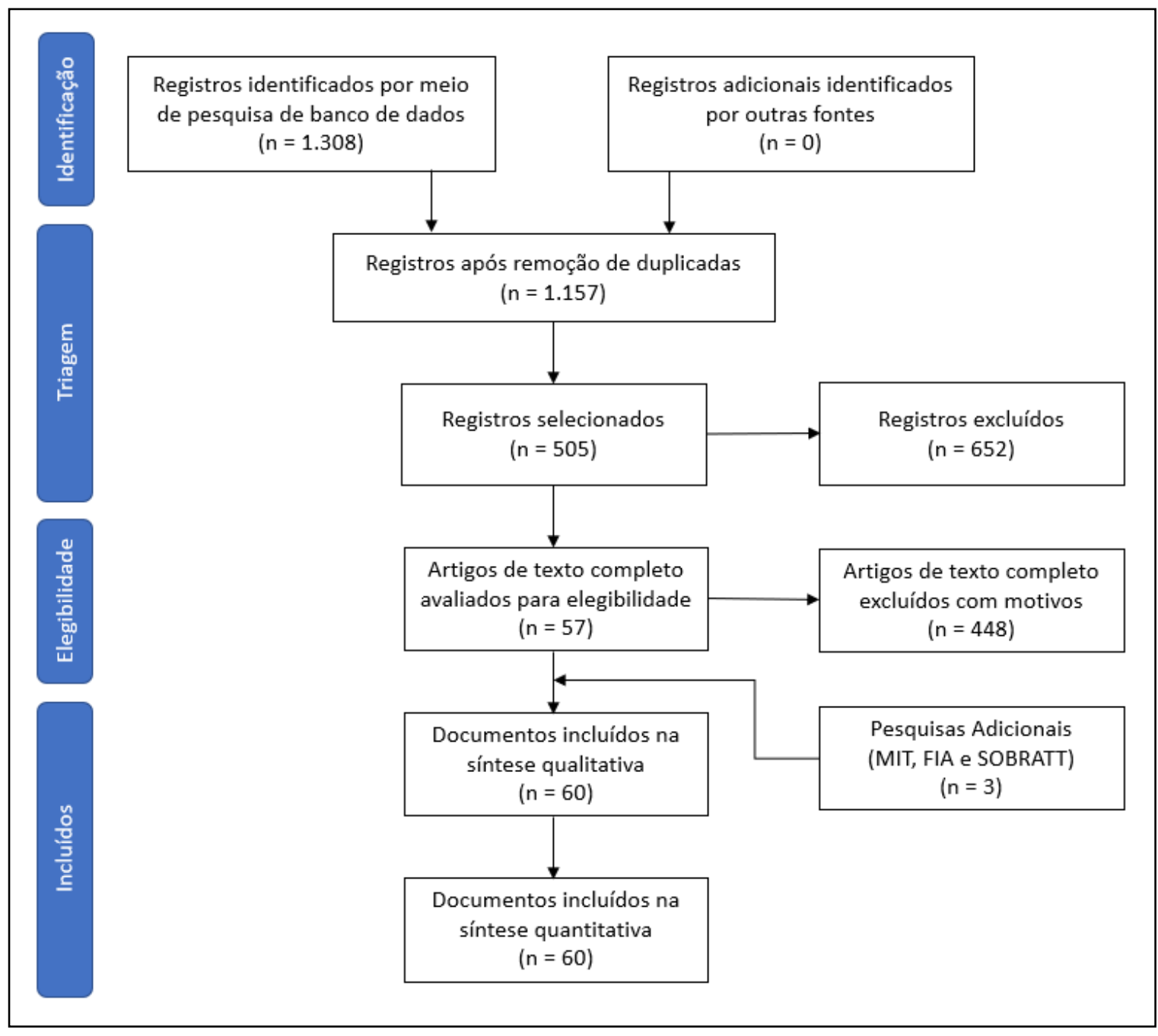

Fonte: Adaptado de Moher et al. (2015).

A Figura 1 ilustra o fluxograma da revisão sistemática, detalhando cada uma das quatro etapas deste protocolo: identificação, triagem, elegibilidade e documentos incluídos para análise.

$\mathrm{Na}$ etapa identificação, realizou-se a busca de artigos nos bancos de dados do Web of Science, Scopus e Google Scholar, cujos critérios de inclusão estão descritos na Tabela 2 - Critérios de Inclusão da Busca.

Tabela 2 - Critérios de Inclusão da Busca.

\begin{tabular}{l|l}
\hline Critério de Inclusão & \multicolumn{1}{c}{ Detalhamento } \\
\hline Palavras-Chave & (“TELEWORK" OR “TELECOMMUTING” OR “HOME OFFICE” OR "FLEXIBLE \\
\hline WORK”) AND (“IMPLEMENTATION" OR "ROADMAP”).
\end{tabular}

Fonte: Resultado da Pesquisa. 
A busca retornou 1.308 resultados, os quais foram organizados por meio dos softwares EndNote X9 e o Microsoft Excel 2019.

$\mathrm{Na}$ etapa da triagem, foram removidas 151 "duplicidades” e excluídos 652 registros, cujo critério de exclusão foram: "artigos com menos de 20 citações" (641), "artigos em idioma diferente do inglês" (09) e "citações" (02). O detalhamento dos critérios de exclusão desta etapa está descrito na Tabela 3 - Critérios de Exclusão da Busca. Concluída a triagem, resultaram 505 registros selecionados para a próxima etapa.

Tabela 3 - Critérios de Exclusão da Busca.

\begin{tabular}{l|l}
\hline Critério de Exclusão & Detalhamento \\
\hline Atributo & Artigo com menos de 20 citações. \\
\hline Idioma & Artigo em idioma diferente do inglês. \\
\hline Tipo de Documento & Livro, Citações, Teses e Dissertações. \\
\hline
\end{tabular}

Fonte: Resultado da Pesquisa.

Na etapa de elegibilidade, foram excluídos 448 artigos completos, de acordo com os seguintes motivos: "publicações cujo acesso integral não é público" (157) e “conteúdo não alinhado à pesquisa” (291). Foram então selecionados 57 documentos para seguirem para a última etapa.

$\mathrm{Na}$ etapa de documentos incluídos para análise, foram adicionadas mais 3 publicações:

- "Work Anywhere" (MIT Technology Review, 2020).

- "Pesquisa Gestão de Pessoas na Crise Covid-19” (FIA, 2020).

- “Pesquisa Home Office 2020” (SOBRATT, 2021).

Foi realizada a leitura integral e criteriosa de todos os documentos e as 60 publicações foram mantidas para a realização das análises quantitativas e qualitativas.

Utilizou-se a análise de conteúdo para complementar de forma objetiva a identificação das abordagens e estratégias das pesquisas sobre a implantação do teletrabalho. Salienta-se seu caráter social, dado que é uma técnica com o objetivo de produzir inferências de um texto para seu contexto social de forma objetiva (Bauer \& Gaskell, 2017).

A análise de conteúdo contempla as seguintes etapas: a) ler de forma integral e minuciosa os documentos selecionado; b) codificar e estruturar as categorias de análise; c) recortar os textos em unidades de registro (palavras, frases, parágrafos) comparáveis e com mesmo conteúdo semântico; d) estabelecer categorias que se diferenciam, tematicamente, nas unidades de registro (passar de dados brutos para dados organizados); e) agrupar as unidades de registro em categorias comuns; f) agrupar progressivamente as categorias (iniciais, intermediárias e finais) g) inferir e interpretar os textos, com base no referencial teórico (Bardin, 1977).

Com base no portfólio de 60 documentos, foi realizada esta análise, cujo resultado será detalhado no próximo tópico deste artigo.

\section{Resultados e Discussão}

Com base nos 60 documentos selecionados, foi realizada inicialmente uma avaliação quanto a volumetria e a distribuição dos países de origem e das regiões geográficas das pesquisas que se referem a implantação do teletrabalho no mundo.

A Figura 2 demonstra a quantidade de publicações por ano sobre implantação do teletrabalho, ao longo do período 
estudado.

Figura 2 - Quantidade de Publicações por Ano.

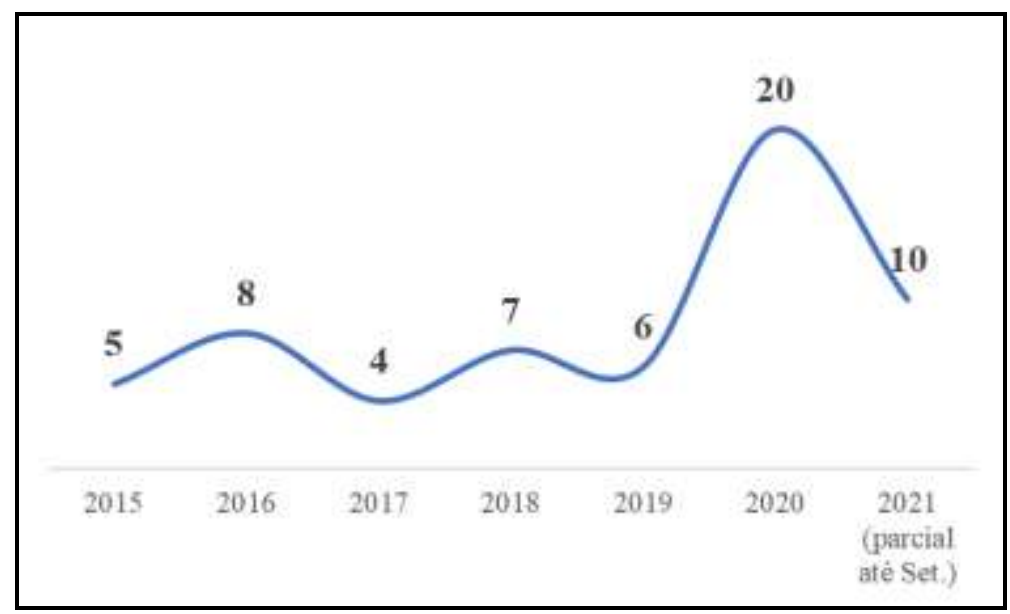

Fonte: Resultado da Pesquisa.

Nota-se que o tema apresenta recorrência em quantidade de publicações, sendo que em 2020 ocorre um pico de publicações de artigos, onde pode-se inferir que o maior interesse pelo tema foi motivado pela deflagração da pandemia em 2020, onde as organizações foram obrigadas a se adaptar à nova realidade mundial.

Em complemento a visão acima, a Figura 3 apresenta a distribuição da quantidade de publicações por país de origem.

Nota-se que a implantação do teletrabalho vem sendo amplamente discutido por muitos países ao redor do mundo. Os Estados Unidos é o país que lidera a quantidade de publicações sobre o tema (9), seguido da Alemanha e do Brasil, ambos com 5 publicações cada.

Figura 3 - Distribuição das Publicações por País de Origem.

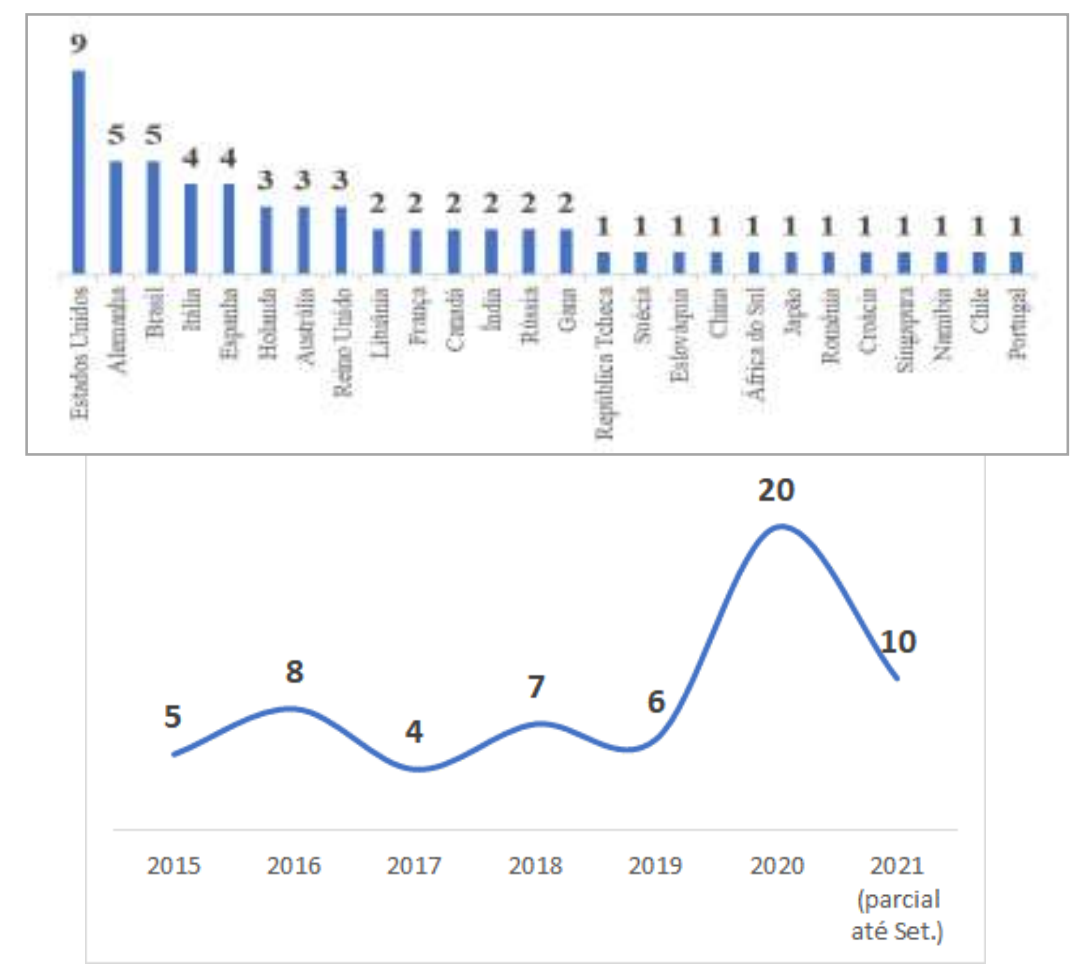

Fonte: Resultado da Pesquisa. 
Ao observar a quantidade de publicações por região geográfica, chega-se ao resultado apresentado na Figura 4 Distribuição das Publicações por Região Geográfica.

Figura 4 - Distribuição das Publicações por Região Geográfica.

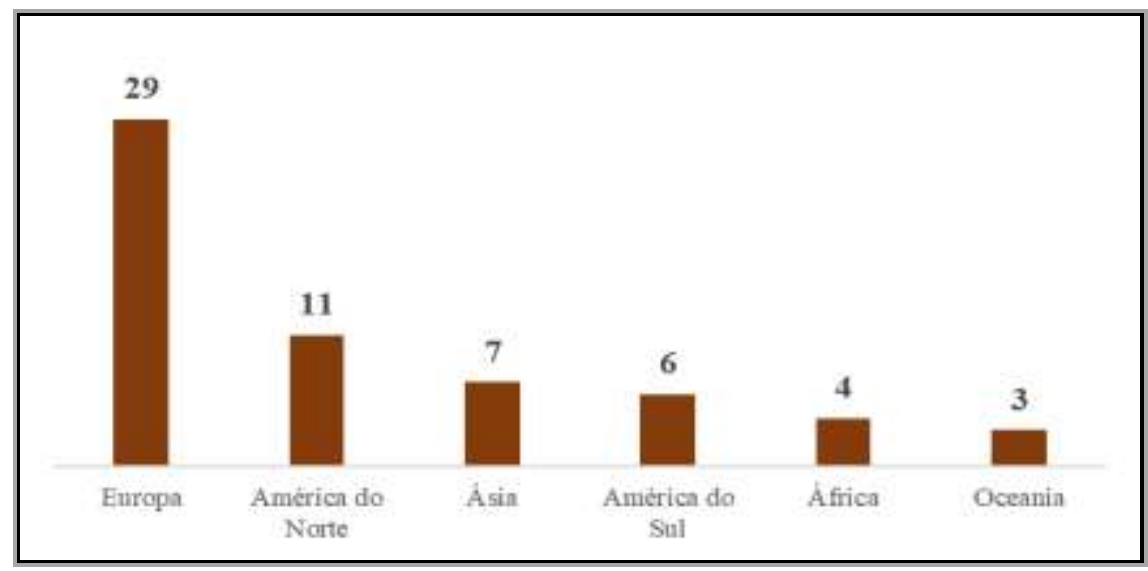

Fonte: Resultado da Pesquisa.

O resultado demonstra que a Europa concentra a maior quantidade de publicações no período estudado, seguido pela América do Norte especialmente por conta dos Estados Unidos.

Considerando os 60 documentos selecionados nesta pesquisa, foi realizada a análise qualitativa por meio do método de análise de conteúdo, cuja evolução das categorias (iniciais, intermediárias e finais) está descrita na Tabela 4 - Evolução das Categorias das Pesquisas sobre Implantação do Teletrabalho.

A Tabela 4 apresenta como categorias finais o resultado de 4 grandes abordagens sobre a implantação do teletrabalho: "Vantagens e Desvantagens" (40), "Estratégia e Práticas de Implantação do Teletrabalho" (10), "Viabilidade" (8) e "Regulamentação" (2).

As publicações que se referem às "Vantagens e Desvantagens" da implantação do teletrabalho se aprofundam sobre o tema basicamente sob 3 aspectos:

- Efeitos no teletrabalhador

- Efeitos do teletrabalho de forma geral

- O teletrabalho como ferramenta de inclusão social 
Research, Society and Development, v. 10, n. 16, e172101623692, 2021

(CC BY 4.0) | ISSN 2525-3409 | DOI: http://dx.doi.org/10.33448/rsd-v10i16.23692

Tabela 4 - Evolução das Categorias das Pesquisas sobre Implantação do Teletrabalho.

\begin{tabular}{|c|c|c|c|c|c|}
\hline Categoria Inicial & Qtde. & Categoria Intermediária & Qtde. & Categoria Final & Qtde. \\
\hline 01. Saúde do Teletraballhador & 5 & \multirow{13}{*}{ I - Efeitos no Teletraballhador } & \multirow{13}{*}{20} & \multirow{29}{*}{$\begin{array}{l}\text { 1- Vantagens e } \\
\text { Desvantagens }\end{array}$} & \multirow{29}{*}{38} \\
\hline 02. Equilibrio entre Vida Pessoal e Profissional & 3 & & & & \\
\hline 03. Interferências, Comunicaçāo Ineficaz e Solidīo & 2 & & & & \\
\hline 04. Saude Mental de Professores: & 1 & & & & \\
\hline 05. Stress pela Falta de Informaçäo e Isolamento & 1 & & & & \\
\hline 06. Technostress & 1 & & & & \\
\hline 07. Exposiçlo ao Ruido & 1 & & & & \\
\hline 08. Atitude, Qualidade de Vida e Saúde & 1 & & & & \\
\hline 09, Produtividade e Qualidade de Vida & 1 & & & & \\
\hline 10. Implantaçào compulsória do Teletrabalho na Pandemia & 1 & & & & \\
\hline 11. Teletraballho por Escolha e por Necessidade (Pandemia) & 1 & & & & \\
\hline 12. Percepçäo de Trabalhadores e Gestores & 1 & & & & \\
\hline 13. Orzanização do Trabalho e Efeitos no Teletrabalhador & 1 & & & & \\
\hline 14. Local e Horáno Flexiveis & 3 & \multirow{12}{*}{ II - Efeitos do Teletrabalho } & \multirow{12}{*}{14} & & \\
\hline 15. Teletraballho em Escolas da França na Pandemia & 1 & & & & \\
\hline 16. Sequrança do Traballhador e Continuidade de Negócio & 1 & & & & \\
\hline 17. Aumento de Produtividade e Reduçio de Custo & 1 & & & & \\
\hline 18. Reduçio de Viagens e Locomoclio & 1 & & & & \\
\hline 19. Futuro do Trabalho Remoto & 1 & & & & \\
\hline 20. Gestäo de Pessoas na Pandemia & 1 & & & & \\
\hline 21. Resultados do Home Office na Pandemia & 1 & & & & \\
\hline 22. Revisào da Literatura quanto a Abordagem das Pesquisas & 1 & & & & \\
\hline 23. Sustentabilidade Económica, Ambiental e Social & 1 & & & & \\
\hline 24. Indicadores de Sustentabilidade & 1 & & & & \\
\hline 25. Perfil Profissional e Condigöes de Trabalho & 1 & & & & \\
\hline 26. Inclusajo Social de Pessoas com Deficiencia & 1 & \multirow{4}{*}{ III - Inclusiào Social } & \multirow{4}{*}{4} & & \\
\hline 27. Teletraballho para a Mão-de-Obra Jovem & 1 & & & & \\
\hline 28. Parn Pessoas com Deficiéncia ou Reabilitaclio & 1 & & & & \\
\hline 29. Para Mulheres em Reabilitaçto Pós-Parto & 1 & & & & \\
\hline 30. Modelo de Liderança para Teletrabalho & 1 & \multirow{4}{*}{ IV - Politicas de Gestáo de Pessoas } & \multirow{4}{*}{4} & \multirow{9}{*}{$\begin{array}{l}\text { II - Estrategia e } \\
\text { Priticas de } \\
\text { Implantaçăo do } \\
\text { Teletraballho }\end{array}$} & \multirow{9}{*}{11} \\
\hline 31. Proposta para Politicas de Teletraballho & 1 & & & & \\
\hline 32. Implantaçà̀o nos Serviços Püblico e Privado Americano & 1 & & & & \\
\hline 33. Abordagenn do Trabalhador para Oferta do Teletraballho & 1 & & & & \\
\hline 34. Fatores Tecnológicos, Ambientais e Organizacionais & 2 & \multirow{2}{*}{ V-Critérios de Adoçalo } & \multirow{2}{*}{3} & & \\
\hline 35. Teenologias Digitais e Priticas Formais de Teletraballho & 1 & & & & \\
\hline 36. Cybersecurity e Engenharia Social & 2 & VI - Pratica de Cybersecurity & 2 & & \\
\hline 37. Flexibilização e Virtualizaç̃o da Organizaçio & 1 & \multirow{2}{*}{ VII - Design da Virtunlizaçẫo } & \multirow{2}{*}{2} & & \\
\hline 38. Modelo de ajuste de teletrabalho induzido por epidemia & 1 & & & & \\
\hline 39. Motivaclio da Empresa para o Teletrabalho & 1 & \multirow{5}{*}{ VIII - Barreiras e Facilitadores } & \multirow{5}{*}{5} & \multirow{8}{*}{ III - Vabilidade } & \multirow{8}{*}{9} \\
\hline 40. Motivaçìo do Traballhador para o Teletrabalho & 1 & & & & \\
\hline 41. Oferta do Teletrabalho versus Engajamento & 1 & & & & \\
\hline 42. Relaça dos Valores Culturais e Coutrole de Tarefas & 1 & & & & \\
\hline 43. Trabalho Colaborativo & 1 & & & & \\
\hline 44. Perfil do Teletrabalhador Europeu & 2 & \multirow{2}{*}{ LX - Perfil do Teletraballhador } & & & \\
\hline 45. Fatores para Elegibitidade do Teletraballhador & 1 & & 3 & & \\
\hline 46. Empregos Teletrabalhiveis na Europa & 1 & X-Asaliaça do Tipo de Trabalho & 1 & & \\
\hline 47. Implantacão no Servico Público Brasileiro & 1 & XI - Legislaçäo Brasileira & 1 & & \\
\hline 48. Nova Legislacialo Russa sobre Teletraballho & 1 & XII - Legislactio Russa & 1 & Regulamentaç3o & 2 \\
\hline Total & 60 & Total & 60 & Total & 60 \\
\hline
\end{tabular}

Fonte: Resultado da Pesquisa.

A Tabela 5 detalha as publicações que se referem aos efeitos da implantação do teletrabalho no teletrabalhador. 
Research, Society and Development, v. 10, n. 16, e172101623692, 2021

(CC BY 4.0) | ISSN 2525-3409 | DOI: http://dx.doi.org/10.33448/rsd-v10i16.23692

Tabela 5 - Publicações por Tipo de Efeito no Teletrabalhador.

\begin{tabular}{|c|c|c|c|}
\hline $\begin{array}{l}\text { Tipo de Efeito no } \\
\text { Teletrabalhador }\end{array}$ & Titulo & Autor & Citaçóes \\
\hline \multirow{9}{*}{$\begin{array}{l}\text { Saúde do } \\
\text { Teletrabalhador } \\
\text { (saúde mental, stress, } \\
\text { technostress, } \\
\text { exposiçāo ao ruido, } \\
\text { etc) }\end{array}$} & $\begin{array}{l}\text { Wellbeing costs of technology use during covid-19 remote working: an } \\
\text { investigation using the ltalian translation of the technostress creators scale }\end{array}$ & (Molino et al., 2020) & 87 \\
\hline & $\begin{array}{l}\text { Case study: A survey of perceived noise in Canadian multi-unit residential } \\
\text { buildings to study long-term implications for widespread teleworking }\end{array}$ & $\begin{array}{l}\text { (Andargie, Tonchie \& } \\
\text { O'Brien, 2021) }\end{array}$ & 64 \\
\hline & Mental bealth of teachers who have teleworked due to covid-19 & $\begin{array}{l}\text { (Palma-Vasquez, Carrasco } \\
\text { \& Hernando-Rodriguez, } \\
\text { 2021) }\end{array}$ & 52 \\
\hline & $\begin{array}{l}\text { Why are teleworkers stressed? An empirical analysis of the causes of telework- } \\
\text { enabled stress }\end{array}$ & $\begin{array}{l}\text { (Weinert, Maier \& } \\
\text { Laumer, 2015) }\end{array}$ & 52 \\
\hline & $\begin{array}{l}\text { A rapid review of mental and physical health effects of working at home: how } \\
\text { do we optimise health? }\end{array}$ & $\begin{array}{l}\text { (Oakman, Kinsman, } \\
\text { Stuckey, Graham \& } \\
\text { Weale, 2020) }\end{array}$ & 51 \\
\hline & $\begin{array}{l}\text { Supporting interdependent telework employees: A moderated-mediation model } \\
\text { linking daily COVID-19 task setbacks to next-day work withdrawal. }\end{array}$ & $\begin{array}{l}\text { (Chong, Huang \& Chang. } \\
\text { 2020) }\end{array}$ & 50 \\
\hline & $\begin{array}{l}\text { The costs and benefits of home office during the covid-19 pandemic; Evidence } \\
\text { from infections and an input-output model for germany }\end{array}$ & $\begin{array}{l}\text { (Fadinger \& Schymik, } \\
\text { 2020) }\end{array}$ & 44 \\
\hline & $\begin{array}{l}\text { Anticipated bealth effects and proposed countermeasures following the } \\
\text { immediate introduction of telework in response to the spread of COVID-19: } \\
\text { The findings of a rapid bealth impact assessment in Japan }\end{array}$ & (Nagata et al., 2021) & 22 \\
\hline & $\begin{array}{l}\text { Psychological impacts of the New Ways of Working (NWW): A systematic } \\
\text { review }\end{array}$ & (Kotera \& Vione, 2020) & 22 \\
\hline \multirow{9}{*}{$\begin{array}{l}\text { Equilibrio eutre Vida } \\
\text { Pessoal e } \\
\text { Profissional. } \\
\text { Qualidade de Vida, } \\
\text { Aumento de } \\
\text { Produtividade }\end{array}$} & $\begin{array}{l}\text { Effects of new ways of working on work hours and work location, health and } \\
\text { job-related outcomes }\end{array}$ & $\begin{array}{l}\text { (Nijp, Beckers, Van de } \\
\text { Voorde, Geurts \& } \\
\text { Kompier, 2016) }\end{array}$ & 90 \\
\hline & $\begin{array}{l}\text { Characterization of home working population during COVID-19 emergency: a } \\
\text { cross-sectional analysis }\end{array}$ & (Moretti et al., 2020) & 58 \\
\hline & $\begin{array}{l}\text { Sustainable social and individual implications of telework: A new insight into } \\
\text { the Romanian labor market }\end{array}$ & $\begin{array}{l}\text { (Dima, Tuclea, Vrànceanu } \\
\text { \& Tigu, 2019) }\end{array}$ & 48 \\
\hline & Implementing Smart Working in Public Administration: a follow up study & (Di Tecco et al., 2021) & 40 \\
\hline & $\begin{array}{l}\text { Perceptions of flexible work arrangements in selected African countries during } \\
\text { the coronavirus pandemic }\end{array}$ & $\begin{array}{l}\text { (Atikn, Jeremiah \& } \\
\text { Boateng, 2020) }\end{array}$ & 37 \\
\hline & Enforced remote working and the work-life interface during lockdown & $\begin{array}{l}\text { (Anderson \& Kelliher, } \\
\text { 2020) }\end{array}$ & 33 \\
\hline & $\begin{array}{l}\text { O teletrabalho na percepçto dos teletraballhadores e seus gestores: vantagens e } \\
\text { desvantagens em um estudo de caso }\end{array}$ & (Hau \& Todescat, 2018) & 28 \\
\hline & $\begin{array}{l}\text { Flexible work arrangements and organizational performance-The difference } \\
\text { between employee and employer-driven practices }\end{array}$ & (Klindzic \& Maric, 2019) & 24 \\
\hline & $\begin{array}{l}\text { The increased use of information and communication technology (ICT) among } \\
\text { employees: Implications for work-life interaction }\end{array}$ & (Wet \& Koekemoer, 2016) & 21 \\
\hline \multirow{2}{*}{$\begin{array}{l}\text { Interferència Trabalho- } \\
\text { Casa, Comunicaçăo } \\
\text { Ineficaz e Solidđ̃o }\end{array}$} & $\begin{array}{l}\text { Achieving effective remote working during the COVID-19 pandemic: A work } \\
\text { design perspective }\end{array}$ & $\begin{array}{l}\text { (Wang, Liu, Qian \& } \\
\text { Parker, 2021) }\end{array}$ & 129 \\
\hline & $\begin{array}{l}\text { Workplace responses to COVID-19 associated with mental health and work } \\
\text { performance of employees in Japan }\end{array}$ & $\begin{array}{l}\text { (Sasaki, Kuroda, Tsuno \& } \\
\text { Kawakami, 2020) }\end{array}$ & 59 \\
\hline
\end{tabular}

Fonte: Resultado da Pesquisa.

Os artigos que abordam as "Vantagens e Desvantagens" sob o aspecto dos "Efeitos no Teletrabalhador" aprofundamse em temas relacionados à saúde do teletrabalhador, equilíbrio entre vida pessoal e profissional, qualidade de vida, produtividade, interferência trabalho-casa, comunicação e solidão.

A Tabela 6 detalha as publicações que se referem aos efeitos da implantação do teletrabalho de forma geral. 
Tabela 6 - Publicações por Tipo de Efeito do Teletrabalho.

\begin{tabular}{|c|c|c|c|}
\hline $\begin{array}{l}\text { Tipo de Efeito do } \\
\text { Teletrabalho }\end{array}$ & Título & Autor & Citações \\
\hline \multirow{3}{*}{$\begin{array}{l}\text { Local e Horário } \\
\text { Flexíveis }\end{array}$} & Alternative work arrangements: Two images of the new world of work & $\begin{array}{l}\text { (Spreitzer, Cameron \& } \\
\text { Garret, 2017) }\end{array}$ & 348 \\
\hline & $\begin{array}{l}\text { Are you attracted? Do you remain? Meta-analytic evidence on flexible work } \\
\text { practices }\end{array}$ & $\begin{array}{l}\text { (Onken-Menke, Nuesch \& } \\
\text { Kroll, 2018) }\end{array}$ & 30 \\
\hline & $\begin{array}{l}\text { Telecommuting: The work anywhere, anyplace, anytime organization in the } 21 \mathrm{st} \\
\text { century }\end{array}$ & $\begin{array}{l}\text { (Narayanan, Menon, } \\
\text { Plaisent \& Bernard, 2017) }\end{array}$ & 27 \\
\hline \multirow{3}{*}{$\begin{array}{l}\text { Segurança do } \\
\text { Teletrabalhador e } \\
\text { Continuidade do } \\
\text { Negócio }\end{array}$} & How effective is telecommuting? Assessing the status of our scientific findings & $\begin{array}{l}\text { (Allen, Golden \& } \\
\text { Shockley, 2015) }\end{array}$ & 691 \\
\hline & Teleworking in the Context of the Covid-19 Crisis & $\begin{array}{l}\text { (Belzunegui-Eraso \& Erro- } \\
\text { Garcés, 2020) }\end{array}$ & 272 \\
\hline & $\begin{array}{l}\text { Expected impact of school closure and telework to mitigate COVID-19 } \\
\text { epidemic in France }\end{array}$ & $\begin{array}{l}\text { (Domenico, Pullano, } \\
\text { Coletti, Hens \& Colizza, } \\
2020)\end{array}$ & 48 \\
\hline \multirow{2}{*}{$\begin{array}{l}\text { Sustentabilidade } \\
\text { Econômica, } \\
\text { Ambiental e Social }\end{array}$} & $\begin{array}{l}\text { The role of remote working in smart cities: Lessons learnt from COVID-19 } \\
\text { pandemic }\end{array}$ & (Kylili et al., 2020) & 22 \\
\hline & Implementation of Flexible Work Arrangements for Sustainable Development & $\begin{array}{l}\text { (Ciarniene, Vienazindiene } \\
\text { \& Adamoniene, 2018) }\end{array}$ & 40 \\
\hline $\begin{array}{l}\text { Redução de Viagens e } \\
\text { Locomoção }\end{array}$ & $\begin{array}{l}\text { Working from home: Modeling the impact of telework on transportation and } \\
\text { land use }\end{array}$ & (Moeckel, 2017) & 39 \\
\hline $\begin{array}{l}\text { Perfil Profissional e } \\
\text { Condições de } \\
\text { Trabalho em Serviços } \\
\text { Sociais }\end{array}$ & Telework and Social Services in Spain during the COVID-19 Pandemic & $\begin{array}{l}\text { (Morilla-Luchena, Muñoz- } \\
\text { Moreno, Chaves-Montero } \\
\text { \& Vázquez-Aguado, 2021) }\end{array}$ & 39 \\
\hline $\begin{array}{l}\text { Aumento de } \\
\text { Produtividade e } \\
\text { Redução de Custo } \\
\end{array}$ & $\begin{array}{l}\text { Teleworking as an inducer of productivity increases and cost rationalization: an } \\
\text { empirical application in the Ministry of Justice and Public Security }\end{array}$ & $\begin{array}{l}\text { (Nogueira Filho, Oliveira, } \\
\text { Samy \& Nunes, 2020) }\end{array}$ & 26 \\
\hline $\begin{array}{l}\text { Futuro do Trabalho } \\
\text { Remoto }\end{array}$ & Work Anywhere & $\begin{array}{l}\text { (MIT Technology Review, } \\
\text { 2020) }\end{array}$ & - \\
\hline $\begin{array}{l}\text { Gestão de Pessoas na } \\
\text { Crise Covid-19 }\end{array}$ & Pesquisa Gestão de Pessoas na Crise Covid-19 & $(\mathrm{FIA}, 2020)$ & - \\
\hline
\end{tabular}

Fonte: Resultado da Pesquisa.

Os documentos que abordam as "Vantagens e Desvantagens" sob o aspecto dos "Efeitos do Teletrabalho" de forma geral aprofundam-se em temas relacionados ao futuro do trabalho remoto, local e horário flexível, segurança do teletrabalhador, continuidade do negócio, redução de viagens e locomoção, redução de custos, entre outros.

Ainda na abordagem de "Vantagens e Desvantagens, a Tabela 7 detalha as publicações que se referem ao teletrabalho como ferramenta de inclusão social. 
Tabela 7 - Publicações sobre Teletrabalho como Ferramenta de Inclusão Social

\begin{tabular}{|c|c|c|c|}
\hline $\begin{array}{l}\text { Tipo de } \\
\text { Inclusão Social }\end{array}$ & Título & Autor & Citações \\
\hline \multirow{2}{*}{$\begin{array}{l}\text { Para Inclusão de } \\
\text { Pessoas com } \\
\text { Deficiência }\end{array}$} & $\begin{array}{l}\text { A 'new normal' following COVID-19 and the economic crisis: Using systems } \\
\text { thinking to identify challenges and opportunities in disability, telework, and } \\
\text { rehabilitation }\end{array}$ & $\begin{array}{l}\text { (Jesus, Landry \& Jacobs, } \\
\text { 2020) }\end{array}$ & 83 \\
\hline & $\begin{array}{l}\text { New business structures creating organizational opportunities and challenges } \\
\text { for work disability prevention }\end{array}$ & (Ekberg et al., 2016) & 26 \\
\hline $\begin{array}{l}\text { Para Mulheres em } \\
\text { Reabilitação pós-parto }\end{array}$ & $\begin{array}{l}\text { Women's employment patterns after childbirth and the perceived access to and } \\
\text { use of flexitime and teleworking }\end{array}$ & $\begin{array}{l}\text { (Chung \& Van Der Horst, } \\
\text { 2018) }\end{array}$ & 121 \\
\hline $\begin{array}{l}\text { Para Mão-de-obra } \\
\text { Jovem }\end{array}$ & Remote employment as a form of labor mobility of today's youth & $\begin{array}{l}\text { (Absalyamova \& } \\
\text { Absalyamov, 2015) }\end{array}$ & 29 \\
\hline
\end{tabular}

Fonte: Resultado da Pesquisa.

Os artigos que abordam as "Vantagens e Desvantagens" sob o aspecto do uso do teletrabalho como "Ferramenta para Inclusão Social" aprofundam-se em temas relacionados à inclusão de pessoas com deficiência, de mulheres em reabilitação pós-parto e da mão-de-obra jovem.

A Tabela 8 detalha os artigos que se referem à "Estratégia e Práticas de Teletrabalho".

Tabela 8 - Publicações sobre "Estratégia e Práticas de Teletrabalho".

\begin{tabular}{|c|c|c|c|}
\hline $\begin{array}{l}\text { Praticas de } \\
\text { Teletrabalho }\end{array}$ & Titalo & Autor & Citacoes \\
\hline \multirow{4}{*}{$\begin{array}{l}\text { Politicas de Gestalo de } \\
\text { Pessoas }\end{array}$} & $\begin{array}{l}\text { How to achieve sustainable efficiency with teleworkers: Leadership model in } \\
\text { telework }\end{array}$ & $\begin{array}{l}\text { (Wojcak, Bajzikova, } \\
\text { Sajgalikova \& Polakova. } \\
\text { 2016) }\end{array}$ & 50 \\
\hline & $\begin{array}{l}\text { An orchestrated negotiated exchange: Trading home-based telework for } \\
\text { intensified work }\end{array}$ & $\begin{array}{l}\text { (Bathini \& Kandathil, } \\
\text { 2019) }\end{array}$ & 35 \\
\hline & $\begin{array}{l}\text { Does organization sector matter in leading teleworker teams? A comparative } \\
\text { case study }\end{array}$ & (Burbach \& Day, 2015) & 24 \\
\hline & Telework is work: Navigating the new normal & (Brumana, 2016) & 20 \\
\hline \multirow{3}{*}{$\begin{array}{l}\text { Critérios de Adoçio do } \\
\text { Teletrabalho }\end{array}$} & $\begin{array}{l}\text { The impact of technology on work in the twenty-first ceatury: Exploring the } \\
\text { smart and dark side }\end{array}$ & $\begin{array}{l}\text { (Holland \& Bardoel. } \\
\text { 2016) }\end{array}$ & 80 \\
\hline & Organisational adoption of telecommuting: Evidence from a developing country & (Ansong \& Boateng. 2018) & 37 \\
\hline & Digital technology use during COVTD-19 pandemic: A rapid review & $\begin{array}{l}\text { (Vargo, Zhin, Benwell \& } \\
\text { Yan, 2021) }\end{array}$ & 27 \\
\hline \multirow{2}{*}{$\begin{array}{l}\text { Praticas de } \\
\text { Cybersecurity para } \\
\text { Teletraballo }\end{array}$} & $\begin{array}{l}\text { Sone cyber securify lyygienic protocols for teleworkers in COVID-19 } \\
\text { pandemic period and beyond }\end{array}$ & (Abukari \& Bankas, 2020) & 25 \\
\hline & $\begin{array}{l}\text { Guide to enterprise telework, remote access, and bring your own device } \\
\text { (BYOD) security }\end{array}$ & $\begin{array}{l}\text { (Souppaya \& Scarfooe, } \\
\text { 2016) }\end{array}$ & 25 \\
\hline \multirow{2}{*}{$\begin{array}{l}\text { Virtualizaçio da } \\
\text { Organizaçio }\end{array}$} & $\begin{array}{l}\text { New ways of working (NWW): work space and cultural change in virtualizing } \\
\text { organizations }\end{array}$ & (Kingma, 2019) & 59 \\
\hline & $\begin{array}{l}\text { Adjusting to epidenic-indaced telework: Empirical insights from teleworkers } \\
\text { in France }\end{array}$ & $\begin{array}{l}\text { (Carillo, Cachat-Rosset, } \\
\text { Marsan, Saba \& Klarsfeld, } \\
2021 \text { ) }\end{array}$ & 44 \\
\hline
\end{tabular}

Fonte: Resultado da Pesquisa.

Os artigos que abordam a "Estratégia e Práticas de Teletrabalho" aprofundam-se nas práticas de políticas de gestão de pessoas, de critérios de adoção do teletrabalho, de cyber-security para teletrabalho e de virtualização da organização. 
A Tabela 9 detalha os artigos que se referem à "Viabilidade do Teletrabalho".

Tabela 9 - Publicações sobre a "Viabilidade do Teletrabalho".

\begin{tabular}{|c|c|c|c|}
\hline $\begin{array}{l}\text { Viabilidade do } \\
\text { Teletraballio }\end{array}$ & Titulo & Antor & Citaçōes \\
\hline \multirow{5}{*}{$\begin{array}{l}\text { Barreiras e } \\
\text { Facilitadores }\end{array}$} & $\begin{array}{l}\text { WHY SOME SME'S IN THE CZECH REPUBLIC ADOPT TELEWORK AND } \\
\text { OTHERS NOT? }\end{array}$ & $\begin{array}{l}\text { (Vrchota, Frantikova \& } \\
\text { VIckova, 2019) }\end{array}$ & 70 \\
\hline & A Taxonouy of Employee Motives for Telework & $\begin{array}{l}\text { (Thoupson, Payne, } \\
\text { Alexander, Gaskins \& } \\
\text { Heaning, 2021) }\end{array}$ & 58 \\
\hline & $\begin{array}{l}\text { Firfor telework? Cross-cultural variance and task-control explanations in } \\
\text { oryanizations' formal telework practices }\end{array}$ & $\begin{array}{l}\text { (Peters, Lighart, Bardoel } \\
\text { \& Poutsma, 2016) }\end{array}$ & 34 \\
\hline & $\begin{array}{l}\text { The relationship of workplace flexibility to enployee engagement among } \\
\text { information technology employees in ladia }\end{array}$ & (Ugargol \& Patrick, 2018) & 33 \\
\hline & $\begin{array}{l}\text { Collaborative workplaces for innovation in service conpanies: barriers and } \\
\text { enablers for supporting new ways of working }\end{array}$ & $\begin{array}{l}\text { (Manca, Grijalvo, } \\
\text { Palacios \& Kaulio, 2018) }\end{array}$ & 25 \\
\hline \multirow{3}{*}{$\begin{array}{l}\text { Perfil do } \\
\text { Teletrabalhador }\end{array}$} & $\begin{array}{l}\text { Working from bome- Who is happy? A survey of Lifhuania's enployees during } \\
\text { the COVID-19 quarautine period }\end{array}$ & $\begin{array}{l}\text { (Raisiene, Rapuano, } \\
\text { Varkaleviciuté \& } \\
\text { Stachova, 2020) } \\
\end{array}$ & 103 \\
\hline & $\begin{array}{l}\text { Who is teleworking and where from? Exploring the main determinasts of } \\
\text { telework in Etrope }\end{array}$ & $\begin{array}{l}\text { (López-Iqual \& Rodriguez- } \\
\text { Modroho, 2020) }\end{array}$ & 27 \\
\hline & $\begin{array}{l}\text { Who is eligible for telework? Exploring the fast-growing acceptance of and } \\
\text { ability to telework in Sweden, 2005-2006 to 2011-2014 }\end{array}$ & (Elldér, 2019) & 24. \\
\hline $\begin{array}{l}\text { Atividades: } \\
\text { Teletraballiveis }\end{array}$ & Teleworkability and the COVID-19 crisis: a new digital divide? & $\begin{array}{l}\text { (Sostero, Milasi, Hurley, } \\
\text { Femátodez-Macias \& } \\
\text { Bisello, 2020) }\end{array}$ & 66 \\
\hline
\end{tabular}

Fonte: Resultado da Pesquisa.

Os artigos que abordam a "Viabilidade do Teletrabalho" aprofundam-se sobre as barreiras e facilitadores da implantação do teletrabalho, sobre o perfil do teletrabalhador e sobre as atividades teletrabalháveis.

A Tabela 10 detalha os artigos que se referem à "Regulamentação do Teletrabalho".

Tabela 10 - Publicações sobre a "Regulamentação do Teletrabalho"

\begin{tabular}{l|l|l|l}
\hline $\begin{array}{c}\text { Regulamentação do } \\
\text { Teletrabalho }\end{array}$ & Titulo & Autor & Citações \\
\hline Legislação Brasileira & A aplicação do teletrabalho no serviço público brasileiro & (Da Silva, 2015) & 22 \\
\hline Legislação Russa & $\begin{array}{l}\text { New russian legislation on Employment of teleworkers: Comparative } \\
\text { Assessment and lmplications for Future development }\end{array}$ & $\begin{array}{l}\text { (Gerasimova, Korchumova } \\
\text { \& Chernyaeva, 2017) }\end{array}$ & 22 \\
\hline
\end{tabular}

Fonte: Resultado da Pesquisa.

Os artigos que abordam a "Regulamentação do Teletrabalho" aprofundam-se sobre a legislação brasileira e sobre a legislação russa.

\section{Considerações Finais}

Esta pesquisa sobre roadmaps para implantação do teletrabalho demonstra a relevância deste tema no mundo, com aumento da produção científica especialmente motivada pela pandemia em 2020. O Brasil demonstra importante interesse 
sobre o tema, dado que é o segundo colocado em produção científica juntamente com a Alemanha, atrás apenas dos Estados Unidos. Isto pode ser constatado com a conclusão do objetivo específico que foi a análise quantitativa dos artigos selecionados na revisão sistemática.

Os demais objetivos específicos que foi revisão sistemática da literatura e a análise de conteúdo, atingiram o objetivo principal, que foi responder a questão de pesquisa: quais as abordagens e estratégias de implantação do teletrabalho de pesquisas publicadas entre 2015 e 2021 ?

O resultado geral da pesquisa confirma a proposição P1 onde há abordagens distintas quanto a implantação do teletrabalho e refuta a proposição P2 em que há roadmaps propostos na literatura para apoiar as organizações em como implantar o teletrabalho, dado que não foi localizado nenhum artigo com uma proposta específica de roadmap para implantação do teletrabalho nas pesquisas publicadas desde 2015.

A falta de uma definição comum da nomenclatura utilizada sobre teletrabalho dificulta a quantificação dos estudos e a avaliação da extensão deste tema (Felstead \& Jewson, 2000), sendo que isto se caracteriza como uma limitação desta pesquisa.

A contribuição desta pesquisa é o detalhamento das abordagens das pesquisas sobre a implantação do teletrabalho publicadas entre 2015 e setembro de 2021, para servir de referência a pesquisadores que tem interesse em explorar o tema e suas oportunidades de aprofundamento na literatura.

Dentre as 4 principais abordagens identificadas sobre o teletrabalho, a abordagem predominante se refere as vantagens e desvantagens. Além disso, o fato de não ter sido localizado nenhum artigo com a proposta de roadmap para a implantação do teletrabalho, bem como a pouca quantidade de artigos que se referem a estratégia de implantação desta modalidade de trabalho, sugerem que há lacunas ou oportunidade de aprofundamento neste tipo de abordagem, se apresentando como sugestões para estudos futuros. Outra sugestão é ampliar esta pesquisa, por meio da utilização de outras palavras-chaves que remetem ao teletrabalho.

\section{Referências}

Absalyamova, S. G., \& Absalyamov, T. B. (2015). Remote employment as a form of labor mobility of today's youth. Mediterranean Journal of Social Sciences, 6 (1), 227-227. https://www.richtmann.org/journal/index.php/mjss/article/view/5705.

Abukari, A. M., \& Bankas, E. K. (2020). Some cyber security hygienic protocols for teleworkers in COVID-19 pandemic period and beyond. International Journal of Scientific \& Engineering Research, 11(4), 1401-1407.

Allen, T. D., Golden, T. D., \& Shockley, K. M. (2015). How effective is telecommuting? Assessing the status of our scientific findings. Psychological Science in the Public Interest, 16 (2), 40-68. https://doi.org/10.1177/1529100615593273.

Andargie, M. S., Touchie, M., \& O’Brien, W. (2021). Case study: A survey of perceived noise in Canadian multi-unit residential buildings to study long-term implications for widespread teleworking. SAGE Journals, 28(4) 443-460. https://doi.org/10.1177/1351010X21993742.

Anderson, D., \& Kelliher, C. (2020). Enforced remote working and the work-life interface during lockdown. Gender in Management: An International Journal, 35 (7/8), 677-683. https://doi.org/10.1108/GM-07-2020-0224.

Ansong, E., \& Boateng, R. (2018). Organisational adoption of telecommuting: Evidence from a developing country. The Electronic Journal of Information Systems in Developing Countries, 84(1), e12008. https://doi.org/10.1002/isd2.12008.

Atiku, S. O., Jeremiah, A., \& Boateng, F. (2021). Perceptions of flexible work arrangements in selected African countries during the coronavirus pandemic. South African Journal of Business Management, 51 (1), 10. https://doi.org/10.4102/sajbm.v51i1.2285.

Bardin, L. J. L. (2011). Análise de conteúdo. Almedina.

Baruch, Y., \& King Joan Yuen, Y. (2000), "Inclination to opt for teleworking: A comparative analysis of United Kingdom versus Hong Kong workers", International Journal of Manpower, 21 (7), 521-539. https://doi.org/10.1108/01437720010378980.

Bathini, D. R., \& Kandathil, G. M. (2019). An orchestrated negotiated exchange: Trading home-based telework for intensified work. Journal of Business Ethics, 154(2), 411-423.

Batista, D. A., Galegale, N. V., Azevedo, M. M., \& Feitosa, M. D., (2021). Revisão Sistemática sobre Teletrabalho: A Abordagem das Recentes Pesquisas. Brazilian Journal of Development, 7 (10), 99409-99421. https://doi.org/10.34117/bjdv7n10-316. 
Bauer, M. W., \& Gaskell, G. (2017). Pesquisa qualitativa com texto, imagem e som: um manual prático. Editora Vozes Limitada.

Belzunegui-Eraso, A., \& Erro-Garcés, A. (2020). Teleworking in the Context of the Covid-19 Crisis. Sustainability, 12 (9), 3662. https://doi.org/10.3390/su12093662.

Birkle, C, Pendlebury, D. A., Schnell, J., \& Adams, J. (2020). Web of Science as a data source for research on scientific and scholarly activity. MIT Press Direct. Quantitative Science Studies, 1 (1),363-376. https://doi.org/10.1162/qss_a_00018.

Burbach, M. E., \& Day, F. C. (2015). Does organization sector matter in leading teleworker teams? A comparative case study. International Journal of Business Research and Development, 3 (4), 8-21.

Brumma, F. (2016). Telework is work: Navigating the new normal. Cornell HR Review. https://hdl.handle.net/1813/73011.

Carillo, K., Cachat-Rosset, G., Marsan, J., Saba, T., \& Klarsfeld, A. (2021). Adjusting to epidemic-induced telework: Empirical insights from teleworkers in France. European Journal of Information Systems, 30(1), 69-88. https://doi.org/10.1080/0960085X.2020.1829512.

Chong, S., Huang, Y., \& Chang, C.-H. (D.). (2020). Supporting interdependent telework employees: A moderated-mediation model linking daily COVID-19 task setbacks to next-day work withdrawal. Journal of Applied Psychology, 105 (12), 1408-1422. https://doi.org/10.1037/apl0000843.

Chung, H., \& Van der Horst, M. (2018). Women's employment patterns after childbirth and the perceived access to and use of flextime and teleworking. Human relations, 71(1), 47-72. https://doi.org/10.1177/0018726717713828.

Čiarnienè, R., Vienažindienė, M., \& Adamonienė, R. (2018). Implementation of Flexible Work Arrangements for Sustainable Development. European Journal of Sustainable Development, 7 (4), 11-11. https://doi.org/10.14207/ejsd.2018.v7n4p11.

Da Silva, A. M. S. (2015). The Aplication of Telework in the Brazilian Public Service. Anais do V Congresso Internacional de Direito e Contemporaneidade. http://coral.ufsm.br/congressodireito/anais/2015/1-2.pdf.

De Wet, W., \& Koekemoer, E. (2016). The Increased Use of Information and Communication Technology (ICT) among Employees: Implications for WorkLife Interaction. South African Journal of economic and management sciences, 19 (2), 264-281. https://doi.org/10.4102/sajems.v19i2.1328.

Di Tecco, C. et al. (2021). Implementing Smart Working in Public Administration: a follow up study. La Medicina del Lavoro, 112 (2), 141.

Dima, A. M., Țuclea, C. E., Vrânceanu, D. M., \& Țigu, G. (2019). Sustainable social and individual implications of telework: A new insight into the Romanian labor market. Sustainability, 11 (13), 3506. https://doi.org/10.3390/su11133506.

Di Domenico, L., Pullano, G., Coletti, P., Hens, N., \& Colizza, V. (2020). Expected impact of school closure and telework to mitigate COVID-19 epidemic in France. EPIcx Lab, 15, http://www.epicx-lab.com/covid-19.html.

Ekberg, K. et al. (2016). New business structures creating organizational opportunities and challenges for work disability prevention. Journal of occupational rehabilitation, 26 (4), 480-489. https://doi.org/10.1007/s10926-016-9671-0.

Elldér, E. (2019). Who is eligible for telework? Exploring the fast-growing acceptance of and ability to telework in Sweden, 2005-2006 to 2011-2014. Social Sciences, 8(7), 200. https://doi.org/10.3390/socsci8070200.

Fadinger, H., \& Schymik, J. (2020). The costs and benefits of home office during the covid-19 pandemic: Evidence from infections and an input-output model for Germany. COVID Economics: Vetted and Real-Time Papers, 9, 107-134.

Felstead, A., \& Jewson, N. (1999). In Work, At Home: Towards an Understanding of Homeworking. Routledge. https://doi.org/10.4324/9780203018965.

FIA (2020); Pesquisa Gestão de Pessoas na Crise COVID 19. https://jornal.usp.br/wp-content/uploads/2020/11/Pesquisa-Gestão-de-Pessoas-na-Crise-deCovid-19-ITA.pdf.

Frye, C (2018). Microsoft Excel 2019 step by step. Microsoft Press.

Garfield, E. J. S. (1964). Science Citation Index - A New Dimension in Indexing. Science, 144 (3619). 649-654. http://garfield.library.upenn.edu/essays/v7p525y1984.pdf.

Gerasimova, E., Tatiyana, K., \& Daria, C. (2017). New Russian legislation on Employment of teleworkers: Comparative Assessment and Implications for Future development. Pravo. Zhurnal Vysshey shkoly ekonomiki, (2), 116-129.

Girard, H. (1995). Comprendre le télétravail: un guide pour l'entreprise. Ed. du Téléphone

Hau, F., \& Todescat, M. (2018). Teleworking in the perception of teleworkers and their managers: advantages and disadvantages in a case study. Navus: Revista de Gestão e Tecnología, 8 (3), 37-52.

Holland, P., \& Bardoel, A. (2016). The impact of technology on work in the twenty-first century: Exploring the smart and dark side. The International Journal of Human Resource Management, 27 (21), https://doi.org/10.1080/09585192.2016.1238126.

Hupe, M. (2019). EndNote X9. Journal of Electronic Resources in Medical Libraries, Taylor \& Francis Group, 16 (3-4), 117-119.

Illegems, V., \& Verbeke, A. (2004). Telework: what does it mean for management? Long Range Planning, 37 (4), $319-334$.

Jesus, T. S., Landry, M. D., \& Jacobs, K. (2020). A 'new normal' following COVID-19 and the economic crisis: Using systems thinking to identify challenges and opportunities in disability, telework, and rehabilitation. Work, 67 (1), 37-46. 
Kingma, S. (2019). New ways of working (NWW): workspace and cultural change in virtualizing organizations. Culture and Organization, 25(5), 383-406. https://doi.org/10.1080/14759551.2018.1427747.

Kylili, A. et al. (2020). The role of remote working in smart cities: Lessons learnt from COVID-19 pandemic. Energy Sources, Part A: Recovery, Utilization and Environmental Effects, 1-16. https://doi.org/10.1080/15567036.2020.1831108.

Klindžić, M., \& Marić, M. (2019). Flexible Work Arrangements and Organizational Performance - The Difference between employee and employer - Driven Practices. Društvena istraživanja, 28 (1), 89-108. https://doi.org/10.5559/di.28.1.05.

Kotera, Y., \& Correa Vione, K. (2020). Psychological impacts of the New Ways of Working (NWW): A systematic review. Jornal internacional de pesquisa ambiental e saúde pública, 17 (14), 5080. https://doi.org/10.3390/ijerph17145080.

Leite, A. L., da Cunha Lemos, D., \& Schneider, W. A. (2019). Teletrabalho: uma revisão integrativa da literatura internacional. Contextus - Revista Contemporânea de Economia e Gestão, 17(3), 186-209.

Lopes, S., Costa, M. T., Fernández-Llimós, F., Amante, M. J., \& Lopes, P. F. (2012). A Bibliometria e a Avaliação da Produção Científica: indicadores e ferramentas. In Actas do congresso Nacional de bibliotecários, arquivistas e documentalistas (11).

López-Igual, P., \& Rodríguez-Modroño, P. (2020). Who is teleworking and where from? Exploring the main determinants of telework in Europe. Sustainability, 12(21), 8797. https://doi.org/10.3390/su12218797.

Manca, C., Grijalvo, M., Palacios, M., \& Kaulio, M. (2018). Collaborative workplaces for innovation in service companies: barriers and enablers for supporting new ways of working. Service Business, 12(3), 525-550. https://doi.org/10.1007/s11628-017-0359-0.

MIT (2020). Work Anywhere. MIT Technology Review, Special Edition Home Office, 1 (1).

Moher, D. et al. (2015). Preferred reporting items for systematic review and meta-analysis protocols (PRISMA-P). 4 (1), 1-9. https://doi.org/10.1186/20464053-4-1.

Moeckel, R. (2017). Working from home: Modeling the impact of telework on transportation and land use. Transportation Research Procedia, $26,207-214$.

Molino, M. et al. (2020). Wellbeing Costs of Technology Use during Covid-19 Remote Working: An Investigation Using the Italian Translation of the Technostress Creators Scale. Sustainability, 12(15), 5911. MDPI AG. Retrieved from http://dx.doi.org/10.3390/su12155911.

Moretti, A. et al. (2020). Characterization of home working population during COVID-19 emergency: a cross-sectional analysis. Jornal Internacional de Pesquisa Ambiental e Saúde Pública, 17 (17), 6284. http://doi.org/10.3390/ijerph17176284.

Morgan, R. E. (2004). Teleworking: an assessment of the benefits and challenges. Journal European Business Review, 16 (4), $344-357$. https://doi.org/10.1016/j.trpro.2017.07.021.

Morilla-Luchena, A., Muñoz-Moreno, R., Chaves-Montero, A., \& Vázquez-Aguado, O. (2021). Telework and Social Services in Spain during the COVID-19 Pandemic. Jornal Internacional de Pesquisa Ambiental e Saúde Pública, 18 (2), 725. https://doi.org/10.3390/ijerph18020725.

Nagata, T. et al. (2021). Anticipated health effects and proposed countermeasures following the immediate introduction of telework in response to the spread of COVID-19: The findings of a rapid health impact assessment in Japan. Journal of Occupational Health, 63 (1), e12198. https://doi.org/10.1002/13489585.12198.

Narayanan, L., Menon, S., Plaisent, M., \& Bernard, P. (2017). Telecommuting: The work anywhere, anyplace, anytime organization in the 21st century. Journal of Marketing \& Management, 8 (2), 47-54.

Nijp, H. H., Beckers, D. G., van de Voorde, K., Geurts, S. A., \& Kompier, M. A. (2016). Effects of new ways of working on work hours and work location, health and job-related outcomes. Chronobiology international, 33 (6), 604-618. https://doi.org/10.3109/07420528.2016.1167731.

Nilles, J. M., Carlson, F. R., Gray, P., \& Hanneman, G. (1976). Telecommuting-an alternative to urban transportation congestion. IEEE Transactions on Systems, Man, and Cybernetics, 6 (2), 77-84.

Nogueira Filho, J. D. A., Oliveira, M. A. M., Sämy, F. P. C., \& Nunes, A. (2020). Teleworking as an inducer of productivity increases and cost rationalization: an empirical application in the Ministry of Justice and Public Security. Revista do Serviço Público - RSP, 71 (2), $274-296$. https://doi.org/10.21874/rsp.v71i2.3173.

Oakman, J., Kinsman, N., Stuckey, R., Graham M., \& Weale V. (2020). A rapid review of mental and physical health effects of working at home: how do we optimise health? BMC Public Health, 20, 1825. https://doi.org/10.1186/s12889-020-09875-z.

Onken-Menke, G., Nüesch, S., \& Kröll, C. (2018). Are you attracted? Do you remain? Meta-analytic evidence on flexible work practices. Business Research, 11 (2), 239-277. https://doi.org/10.1007/s40685-017-0059-6.

Palma-Vasquez, C., Carrasco, D., \& Hernando-Rodriguez, J. C (2021). Mental health of teachers who have teleworked due to covid-19. European Journal of Investigation in Health, Psychology and Education, 11 (2), 515-528. http://dx.doi.org/10.3390/ejihpe11020037.

Peters, P., Ligthart, P. E., Bardoel, A., \& Poutsma, E. (2016). 'Fit'for telework'? Cross-cultural variance and task-control explanations in organizations' formal telework practices. The International Journal of human resource management, 27(21), 2582-2603. https://doi.org/10.1080/09585192.2016.1232294.

Raišienè, A. G., Rapuano, V., Varkulevičiūtè, K., \& Stachová, K. (2020). Working from home-Who is happy? A survey of Lithuania's employees during the COVID-19 quarantine period. Sustainability, 12(13), 5332. https://doi.org/10.3390/su12135332. 
Rocha, C. T. M. D., \& Amador, F. S. (2018). O teletrabalho: conceituação e questões para análise. Cadernos Ebape. Br, 16 (1), 152-162. https://doi.org/10.1590/1679-395154516.

Rosenfield, C. L., \& Alves, D. A. (2011). Teletrabalho. In: Cattani, A. D.; Holzmann, L. (Orgs.). Dicionário de trabalho e tecnologia. Porto Alegre: Zouk, 414418 .

Sakuda, L. O., \& Vasconcelos, F. D. C. (2005). Teletrabalho: desafios e perspectivas. Organizações \& Sociedade, 12 (33), 39-49. https://doi.org/10.1590/S1984-92302005000200002.

Sasaki, N., Kuroda, R., Tsuno, K., \& Kawakami, N. (2020). Workplace responses to COVID-19 associated with mental health and work performance of employees in Japan. Journal of Occupational Health, 62 (1), e12134. https://doi.org/10.1002/1348-9585.12134.

Schleder, M. V. N., Gai, M. J. P., De Oliveira, G. C., \& Costa, V. M. F., (2019). Teleworking and Academic Research: Analysis of Scientific Production Available in National Data Banks Opened until 2017. Revista Administração Em Diálogo, 21 (1), 81-98. https://doi.org/10.23925/2178$0080.2017 \mathrm{v} 21 \mathrm{i} 1.39082$.

Silva, A. H., \& Fossá, M. I. T. (2015). Análise de conteúdo: exemplo de aplicação da técnica para análise de dados qualitativos. Qualitas Revista Eletrônica, $16(1)$.

SOBRATT (2020). Home Office - O que muda com o Coronavírus. http://www.sobratt.org.br/index.php/19032020-home-office-o-que- muda-com-ocoronavirus/.

Sostero, M., Milasi, S., Hurley, J., Fernandez-Macias, E., \& Bisello, M. (2020). Teleworkability and the COVID-19 crisis: a new digital divide? JRC working papers series on labour, education, and technology. http://hdl.handle.net/10419/231337.

Souppaya, M., \& Scarfone, K. (2016). Guide to enterprise telework, remote access, and bring your own device (BYOD) security. NIST Special Publication, 800, 46. https://dx.doi.org/10.6028/NIST.SP.800-46r2.

Spreitzer, GM, Cameron, L., \& Garrett, L. (2017). Alternative work arrangements: Two images of the new world of work. Annual Review of Organizational Psychology and Organizational Behavior, 4, 473-499. https://doi.org/10.1146/annurev-orgpsych-032516-113332.

Thompson, R. J., Payne, S. C., Alexander, A. L., Gaskins, V. A., \& Henning, J. B. (2021). A Taxonomy of Employee Motives for Telework. Occupational Health Science, 1-32. https://doi.org/10.1007/s41542-021-00094-5.

Ugargol, J. D., \& Patrick, H. A. (2018). The relationship of workplace flexibility to employee engagement among information technology employees in India. South Asian Journal of Human Resources Management, 5(1), 40-55. https://doi.org/10.1177/2322093718767469.

Van Sell, M., \& Jacobs, SM (1994). Telecommuting and quality of life: a review of the literature and a model for research. Telematics and Informatics, 11 (2), 81-95. https://doi.org/10.1016/0736-5853(94)90033-7.

Vargo, D., Zhu, L., Benwell, B., \& Yan, Z. (2021). Digital technology use during COVID-19 pandemic: A rapid review. Human Behavior and Emerging Technologies, 3(1), 13-24. https://doi.org/10.1002/hbe2.242.

Vrchota, J., Frantíková, Z., \& Vlčková, M. (2019). Why Some SMEs in the Czech Republic Adopt Telework and Others Not? European Countryside, 11(4), $599-615$.

Wang, B., Liu, Y., Qian, J., \& Parker, S. K (2021). Achieving effective remote working during the COVID-19 pandemic: A work design perspective. Applied Psychology, 70 (1), 16-59. https://doi.org/10.1111/apps.12290.

Watad, M. M \& Will, P. C (2003). Telecommuting and organizational change: a middle-managers' perspective. Business Process Management Journal, 9 (4), 459-472. https://doi.org/10.1108/14637150310484517.

Weinert, C., Maier, C., \& Laumer, S. (2015). Why are teleworkers stressed? An empirical analysis of the causes of telework-enabled stress. Wirtschaftsinformatik Proceedings 2015, 1407-1421.

Wojcak, E., Bajzikova, L., Sajgalikova, H., \& Polakova, M. (2016). How to achieve sustainable efficiency with teleworkers: Leadership model in telework. Procedia-Social and Behavioral Sciences, 229, 33-41. https://doi.org/10.1016/j.sbspro.2016.07.111. 